The Astrophysical Journal, 560:L181-L184, 2001 October 20

(C) 2001. The American Astronomical Society. All rights reserved. Printed in U.S.A.

\title{
MILLIMETER-WAVE APERTURE SYNTHESIS IMAGING OF VEGA: EVIDENCE FOR A RING ARC AT 95 AU
}

\author{
D. W. Koerner, ${ }^{1}$ A. I. Sargent, ${ }^{2}$ and N. A. Ostroff ${ }^{1}$ \\ Received 2001 August 14; accepted 2001 September 6; published 2001 September 25
}

\begin{abstract}
We present the first millimeter-wave aperture synthesis map of dust around a main-sequence star. A $3^{\prime \prime}$ resolution image of $1.3 \mathrm{~mm}$ continuum emission from Vega reveals a clump of emission 12" from the star at a position angle (P.A.) of $45^{\circ}$, consistent with the location of maximum $850 \mu \mathrm{m}$ emission in a lower resolution James Clerk Maxwell Telescope/Submillimeter Common-User Bolometric Array (SCUBA) map. The flux density is $4.0 \pm$ $0.9 \mathrm{mJy}$. Adjacent $1.3 \mathrm{~mm}$ peaks with flux densities of $3.4 \pm 1.0$ and $2.8 \pm 0.9 \mathrm{mJy}$ are located $14^{\prime \prime}$ and $13^{\prime \prime}$ from the star at P.A.'s of $67^{\circ}$ and $18^{\circ}$, respectively. An arclike bridge connects the two strongest peaks. There is an additional $2.4 \pm 0.8 \mathrm{mJy}$ peak to the southwest, $11^{\prime \prime}$ from the star at P.A. $=215^{\circ}$, and a marginal detection, $1.4 \pm 0.5 \mathrm{mJy}$, at the stellar position, consistent with photospheric emission. An extrapolation from the $850 \mu \mathrm{m}$ flux, assuming $F_{1.3-0.85 \mathrm{~mm}} \propto \lambda^{-2.8}$, agrees well with the total detected flux for Vega at $1.3 \mathrm{~mm}$ and implies a dust emissivity index $\beta$ of 0.8 We conclude that we have detected all but a very small fraction of the dust imaged by SCUBA in our aperture synthesis map and that these grains are largely confined to segments of a ring of radius $95 \mathrm{AU}$.
\end{abstract}

Subject headings: circumstellar matter — planetary systems: formation -

planetary systems: protoplanetary disks — stars: individual ( $\alpha$ Lyrae)

\section{INTRODUCTION}

The IRAS detection of excess infrared emission from Vega ( $\alpha$ Lyrae) heralded the discovery that dust grains surround many nearby stars (Aumann et al. 1984; Gillett 1986). High-resolution images at wavelengths ranging from the optical to millimeter regimes have since revealed that, in many cases, the dust around similar "Vega-excess" stars is configured in circumstellar disks and rings (Smith \& Terrile 1984; see Koerner 2001 for a review). Some of the more massive disks surround stars with ages of order 10 Myr (e.g., Smith \& Terrile 1984; Jura 1991; Zuckerman \& Becklin 1993a) and are readily explainable as the end stages of protostellar/protoplanetary disks like those around younger T Tauri stars (e.g., Beckwith \& Sargent 1993). The persistence of material in orbit around older stars like Vega (with an age of 350 Myr; Maeder \& Meynet 1988) is more problematic since radiation pressure and Poynting-Robertson drag should have dispersed any grains on much shorter timescales. Traditionally, this conundrum has been resolved by postulating an unseen population of asteroidal or cometary bodies that collisionally regenerate a "debris disk" (e.g., Backman \& Paresce 1993), although particles may also be dynamically trapped in mean motion resonances with larger orbiting bodies (Ozernoy et al. 2000).

Coronagraphic imaging of $\beta$ Pictoris quickly provided a clear picture of its circumstellar disk (Smith \& Terrile 1984). However, it has proved difficult to establish unambiguously the distribution of dust around Vega. The first IRAS observations resolved the $60 \mu \mathrm{m}$ emission in a $25^{\prime \prime}$ beam and indicated a 60-100 $\mu \mathrm{m}$ color temperature of $85 \mathrm{~K}$ (Aumann et al. 1984). Gillett (1986) estimated a size of $29^{\prime \prime} \times 25^{\prime \prime}(\mathrm{FWHM})$ at a position angle (P.A.) of $85^{\circ}$ and postulated the presence of a dust reservoir radially confined between 36 and $170 \mathrm{AU}$ for large grains $(>100 \mu \mathrm{m})$ or between 14 and $400 \mathrm{AU}$ for small grains $(<10 \mu \mathrm{m})$. He also noted that grains smaller than $1.1 \mathrm{~mm}$ should have been depleted by Poynting-Robertson

\footnotetext{
${ }^{1}$ University of Pennsylvania, David Rittenhouse Laboratory, 209 South 33d Street, Philadelphia, PA 19104-6396.

${ }^{2}$ Division of Physics Mathematics and Astronomy, MS 103-33, California Institute of Technology, Pasadena, CA 91125.
}

drag. A reexamination of the original resolved $60 \mu \mathrm{m}$ emission led to a source size of $27^{\prime \prime} \times 27^{\prime \prime}(\mathrm{FWHM})$, implying a radius of $106 \mathrm{AU}$ and grains ranging in size from 0.1 to $10 \mu \mathrm{m}$ (van der Bliek, Prusti, \& Waters 1994). A pole-on configuration was inferred from the circular symmetry and is consistent with the stellar orientation implied by optical spectroscopy (Gulliver, Hill, \& Adelman 1994). Kuiper Airborne Observatory (KAO) measurements by Harvey, Wilking, \& Joy (1984), in 30" and $43^{\prime \prime}$ beams at $\lambda=47$ and $95 \mu \mathrm{m}$, respectively, yielded a lower color temperature, $78 \mathrm{~K}$, than IRAS and suggested that different amounts of material are enclosed by the different beams. Combining these results with measurements in KAO apertures placed 1' away from the star, these authors proposed that the extent of the dust around Vega was as large as 46" (FWHM). Recent ISOCAM observations at 60 and $90 \mu \mathrm{m}$ support this suggestion, yielding Gaussian widths (60\% peak value) of $22^{\prime \prime} \pm 2^{\prime \prime}$ and $36^{\prime \prime} \pm 3^{\prime \prime}$, respectively (Heinrichsen, Walker, \& Klaas 1998). Deconvolution of the $60 \mu \mathrm{m}$ ISOCAM image was found to be consistent with an extended source surrounded by a ring of $42^{\prime \prime}$ diameter. ISOPHOT measurements from 25 to $200 \mu \mathrm{m}$ imply a color temperature of $73 \mathrm{~K}$, a grain emissivity index $\beta=1.1$, where emissivity $Q \propto \lambda^{-\beta}$, and the presence of grains of size $110 \mu \mathrm{m}$ (Heinrichsen et al. 1998).

Initial observations of Vega at submillimeter wavelengths were carried out using beams of diverse sizes that complicated the interpretation (Chini, Krügel, \& Kreysa 1990; Chini et al. 1991; see discussion in Zuckerman \& Becklin 1993b). A sevenpoint map at $\lambda=800 \mu \mathrm{m}$, extending to $16^{\prime \prime}$ from the star, revealed excess emission in an $18^{\prime \prime}$ diameter field $8^{\prime \prime}$ east and 14 " north of the stellar position (Zuckerman \& Becklin 1993b). This first indication of asymmetric morphology also demonstrated that the source extended beyond many of the beams used in early submillimeter-wave measurements.

The asymmetric distribution of dust was dramatically confirmed by Holland et al. (1998, hereafter H98) in their $850 \mu \mathrm{m}$ image of Vega obtained at the James Clerk Maxwell Telescope using the Submillimeter Common-User Bolometric Array (SCUBA). Although extended emission appears circular at the lowest intensity levels in this image, a more linear core oriented 

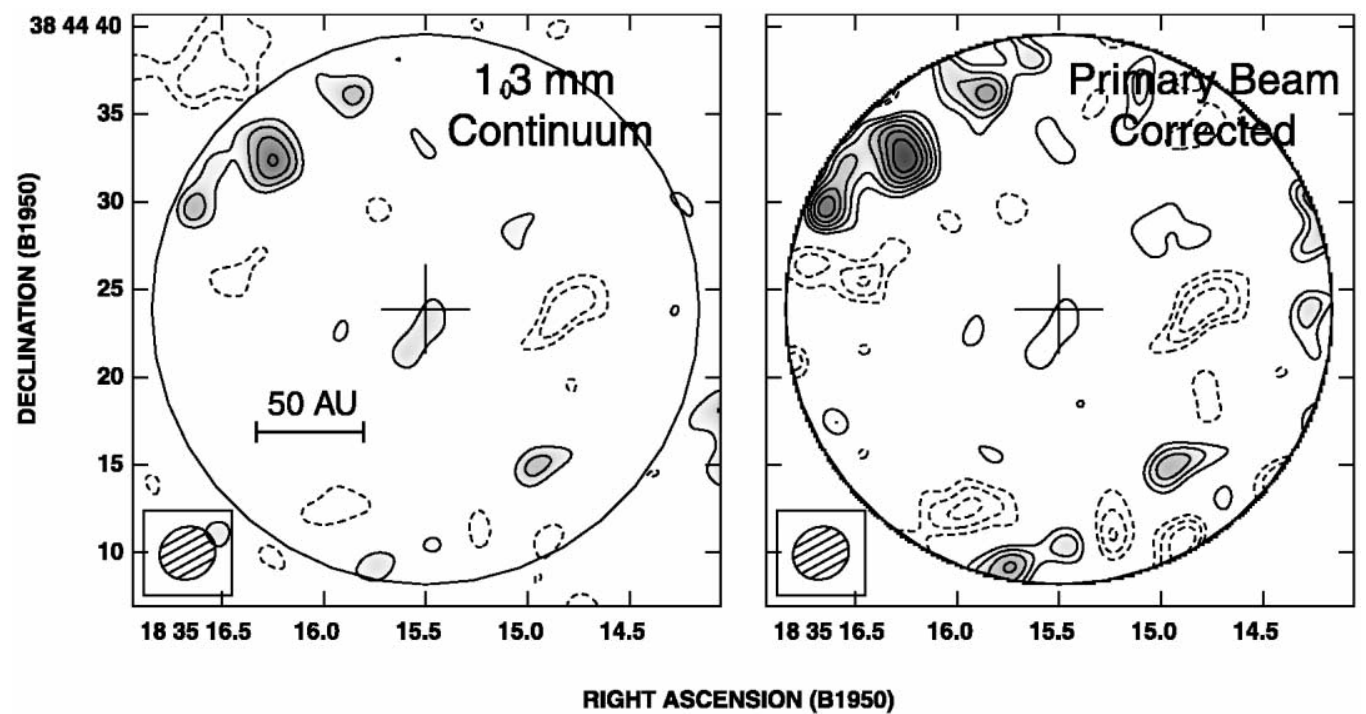

FIG. 1.-Left: Aperture synthesis image of $1.3 \mathrm{~mm}$ emission from Vega ( $\alpha$ Lyrae). Contours are at $1 \sigma$ intervals of $0.5 \mathrm{mJy}$, starting at the $2 \sigma$ level. The position of Vega is marked with a plus sign; the half-power circumference of the primary beam of the $10.4 \mathrm{~m}$ telescopes is shown as a circle. The synthesized beam, 3"3 $\times 2$ 2.9 FWHM at P.A. = $119^{\circ}$, is represented by a hatched circle. Right: Same as the left panel, except that compensation has been made for the radial falloff in intensity due to the primary-beam pattern.

at P.A. $\sim 45^{\circ}$ encompasses a bright peak at $9^{\prime \prime}$ (70 AU) from the estimated position of the star. Combining the SCUBA results with Vega fluxes from $10 \mu \mathrm{m}$ to $1 \mathrm{~mm}$, Dent et al. (2000, hereafter DWHG) find a dust temperature of $80 \mathrm{~K}$, effective grain sizes of $70 \mu \mathrm{m}$, and $\beta=0.8$. Their modeling suggests that the dust is confined to a ring centered on the star with an inner radius of greater than $80 \mathrm{AU}$ and an outer radius of less of $120 \mathrm{AU}$ (i.e., between $10^{\prime \prime}$ and $15^{\prime \prime}$ from the star). The brightness asymmetry seen in the SCUBA maps demands density enhancements of at least a factor of 2 in the northeast sector of the putative ring. With the higher resolution attainable with the Owens Valley Radio Observatory (OVRO) millimeter-wave array, we can image the distribution of dust grains around Vega in greater detail. Here we present 3 " resolution aperture synthesis images of $1.3 \mathrm{~mm}$ emission from Vega that strongly support the pole-on ring hypothesis.

\section{OBSERVATIONS AND RESULTS}

Two low-resolution configurations (with baseline lengths from 15 to $115 \mathrm{~m}$ ) of the six $10.4 \mathrm{~m}$ telescopes of the OVRO millimeter array were used to observe Vega in the $1.3 \mathrm{~mm}$ band in four $1 \mathrm{GHz}$ bands at 229.0, 230.5, 233.5, and $235.0 \mathrm{GHz}$. Observations were made on 15 occasions between the fall of 1999 and the spring of 2001. These observing cycles each lasted from 4 to $10 \mathrm{hr}$ and included 25 minute integrations on Vega interspersed with 10 minute integrations on $3 \mathrm{C} 345$ for phase and amplitude visibility calibrations. The flux density for $3 \mathrm{C}$ 345 was based on measurements of Neptune and Uranus and varied between 4.0 and 4.6 Jy during the course of the project. The phase center of the observations was the optical position of Vega, $\alpha(1950)=8^{\mathrm{h}} 35^{\mathrm{m}} 14.655, \delta(1950)=+38^{\circ} 44^{\prime} 09^{\prime \prime} 68$, modified by proper motions $\Delta \alpha=201 \mathrm{mas} \mathrm{yr}^{-1}$ and $\Delta \delta=$ $285 \mathrm{mas} \mathrm{yr}^{-1}$. These proper motions resulted in a total positional shift of less than 0.5 throughout the observing period. Visibilities were calibrated using Caltech's MMA software and were combined with NRAO's AIPS package. The AIPS task IMAGR was used to make a map that combined natural weighting with a Gaussian taper. A taper with a half-power radius of $80 \mathrm{k} \lambda$ yielded the best signal-to-noise ratio. The resulting synthesized beam size is 3".3 $\times 2.9(\mathrm{FWHM})$ at P.A. $=119^{\circ}$, and the rms noise amplitude is $0.5 \mathrm{mJy}$.

Continuum emission is detected at several locations in the $1.3 \mathrm{~mm}$ image of Vega displayed in the left panel of Figure 1. For each location, the separation from the star and flux density is listed in columns (1)-(3) of Table 1. The brightest peak lies 12 ". $2 \pm 0$ ".6 northeast of the stellar position $(95 \mathrm{AU}$ at the $7.8 \mathrm{pc}$ distance of Vega) and is flanked by two fainter detections. The brighter of these is linked to the main peak by an

TABLE 1

Flux Densities ${ }^{\mathrm{a}}$ IN MaP

\begin{tabular}{|c|c|c|c|c|c|c|}
\hline \multirow{2}{*}{$\begin{array}{l}\text { SEPARATION } \\
\text { (arcsec) } \\
(1)\end{array}$} & \multirow{2}{*}{$\begin{array}{l}\text { P.A. } \\
\text { (deg) } \\
\text { (2) }\end{array}$} & \multirow[b]{2}{*}{$\begin{array}{l}F_{1.3 \mathrm{~mm}} \\
\text { (3) }\end{array}$} & \multirow{2}{*}{$\begin{array}{c}\text { CORRECTED } \\
F_{1.3 \mathrm{~mm}} \\
(4)\end{array}$} & \multicolumn{3}{|c|}{$F_{0.85 \mathrm{~mm}}$} \\
\hline & & & & $\begin{array}{c}\alpha=2 \\
(5)\end{array}$ & $\begin{array}{c}\alpha=3 \\
(6)\end{array}$ & $\begin{array}{l}\text { SCUBA } \\
\text { (7) }\end{array}$ \\
\hline $2.2 \pm 0.6$ & 45 & $2.5 \pm 0.6$ & $4.0 \pm 0.9$ & 9.4 & 14.3 & $17.3 \pm 3$ \\
\hline 4.5 & 6 & & & & 12 & \\
\hline $13.0 \pm 1$. & 18 & $7 \pm$ & $8 \pm 0.9$ & 6. & 10.0 & \\
\hline $11.0 \pm 1.0$ & 215 & $1.7 \pm 0.5$ & $2.4 \pm 0.8$ & 5.6 & 8.6 & $\sim 15$ \\
\hline $0 \ldots$ & $\ldots$ & $1.4 \pm 0.5$ & $1.4 \pm 0.5$ & $5 \pm 1^{b}$ & $5 \pm 1$ & \\
\hline Total & $\ldots$ & $9.1 \pm 1.2$ & $14 \pm 1.9$ & 32.9 & 48.4 & $45.7 \pm 5.4^{\circ}$ \\
\hline
\end{tabular}

${ }^{\mathrm{a}}$ In units of millijanskys.

${ }^{\mathrm{b}}$ Estimated photospheric flux from Vega at $\lambda=850 \mu \mathrm{m}$.

${ }^{\mathrm{c}}$ Integrated emission from map of H98. 
arc of low-intensity emission. The location of the $1.3 \mathrm{~mm}$ maximum is consistent with that of the peak of $850 \mu \mathrm{m}$ emission in a $14^{\prime \prime}$ beam found by H98, 9" $\pm 2^{\prime \prime}$ northeast of the star. An additional weaker source is seen southwest of the star at a distance of $11^{\prime \prime} \pm 1^{\prime \prime}$ (86 AU). Its location and brightness relative to the northeast intensity enhancements are also consistent with the morphology of the SCUBA map. Our marginal detection of radiation at the stellar position, $1.4 \pm 0.5 \mathrm{mJy}$, is compatible with the expected photospheric value, $2.5 \pm$ $1.0 \mathrm{mJy}$, extrapolating from model fluxes at shorter wavelengths (Cohen et al. 1992).

Since the emission peaks in our map are not far from the half-power radius of the primary OVRO array beam, $\theta_{B} / 2=$ $16^{\prime \prime}$, their brightness is underrepresented in the left panel of Figure 1. Correction for this diminution was carried out with AIPS task PBCOR and produced the map displayed in the right panel of Figure 1. The corresponding flux densities are listed in column (4) of Table 1. For comparison with our image, the $850 \mu \mathrm{m}$ fluxes (H98) must be extrapolated with an appropriate relation, $F_{\lambda} \propto \lambda^{-\alpha}$. These extrapolated values are tabulated in columns (5) and (6) of Table 1 for $\alpha=2$ and 3, respectively. Corresponding SCUBA fluxes are listed in column (7). For $\alpha=2$, the fluxes listed in column (4) account for only $75 \%$ of the $850 \mu$ m flux; with $\alpha=3$, the OVRO and SCUBA fluxes agree within the uncertainties.

\section{DISCUSSION}

The 3 " resolution OVRO maps in Figure 1 strongly support the hypothesis that the dust around Vega is distributed in a clumpy ring, as DWHG suggested. Emission appears at distances of 85-110 AU from the star. Its brightness and distribution are consistent with the size and asymmetry of the emission in the $14^{\prime \prime}$ resolution SCUBA map. The brightest $1.3 \mathrm{~mm}$ clump, at $95 \mathrm{AU}$, is connected to a neighboring peak by a lowintensity bridge that forms an arclike structure centered on the star. Dust is also detected on the opposite side of the star at a nearly equidistant position. All the flux from the SCUBA map is recovered in the $1.3 \mathrm{~mm}$ detections, if a plausible emissivity law is adopted. This suggests that there is little broad extended emission. However, our observations do not rule out the presence of material beyond the edge of the OVRO primary beam, $16 "$ (125 AU), as suggested by 95 and $90 \mu \mathrm{m}$ measurements (Harvey et al. 1984; Heinrichsen et al. 1998).

The total flux in the $850 \mu \mathrm{m}$ map of H98, $45.7 \mathrm{mJy}$, implies a $1.3 \mathrm{~mm}$ flux density equal to that of sources detected in our map, $14 \mathrm{mJy}$, if the wavelength dependence of the flux density $F_{0.85-1.3 \mathrm{~mm}} \propto \lambda^{-\alpha}$ has $\alpha=2.8$. H98 report a value of $\alpha=2.7$ for $F_{\lambda}$ between 850 and $1350 \mu \mathrm{m}$ in a single aperture centered on the peak of $850 \mu \mathrm{m}$ emission. For optically thin dust radiating in the Rayleigh-Jeans regime, our best-fit value for $\alpha$ implies a grain emissivity, $Q \propto \lambda^{-\beta}$, with $\beta=0.8$. The same value was derived by DWHG in modeling of fluxes from 10 to $1000 \mu \mathrm{m}$ and, together with the radial location of the dust, implies grain sizes between 30 and $200 \mu \mathrm{m}$.

Taken together, our results are consistent with Vega being surrounded by a ring of highly variable density. From model fitting, both to the image of H98 and to long-wavelength fluxes, DWHG predicted a circum-Vega ring with density enhancements of a factor of 2 in the northeast segment. Emission at levels less than half that of even the strongest peak in the left panel of Figure 1 would be below our $3 \sigma(1.5 \mathrm{mJy})$ detection threshold. As a consequence, a continuous dust ring remains unseen, but the arclike structure of the patchy maxima provide compelling evidence of its presence. The detection of a much weaker, but symmetrically placed, dust continuum peak to the southwest adds to this evidence. Moreover, in the right panel of Figure 1, after correction for the effects of the primary beam, several clumps are detectable at the $2 \sigma$ confidence level. All lie along a possible orbital trajectory for ring material.

There is some evidence for clumping of dust in rings in other extrasolar systems. The narrow ring around HR 4796 (Koerner et al. 1998; Schneider et al. 1999) shows strong evidence of asymmetric brightening at one ansa (Telesco et al. 2000). This feature has been explained as the result of eccentricity forcing, either by a stellar companion or by an unseen planet (Wyatt et al. 1999). An $850 \mu \mathrm{m}$ SCUBA image of $\epsilon$ Eridani shows a still greater degree of azimuthal asymmetry (Greaves et al. 1998). The ring discontinuity presented here is even more pronounced. The images in Figure 1 present a picture that resembles that discovered by stellar occultations of the planet Neptune; partial "ring arcs" were inferred from the fact that occultations were observed only along preferred trajectories (Hubbard et al. 1986). Subsequent Voyager images revealed that the arcs were actually connected by tenuous ribbons of dust (Smith et al. 1989). Theoretical analyses concluded that the material is azimuthally confined by resonant interactions with Neptune's satellite Galatea (Goldreich, Tremaine, \& Borderies 1986; Porco 1991; Hanninen \& Porco 1997). The observations presented here suggest that there may be useful parallels to be drawn between the ring arcs associated with Neptune and the distribution of dust around Vega. Future submillimeter- and millimeter-wave observations, especially with SAO's Submillimeter Array and with the Combined Array for Research in Millimeter Astronomy, should make it possible to determine Vega's dust structure in even greater detail and to infer details of any hidden planetary bodies responsible for the morphology of a ring arc.

We are grateful to all OVRO postdoctoral scholars and visiting astronomers who helped in acquiring the data, but especially to Kartik Sheth. The OVRO millimeter array is supported by the NSF through grant AST 99-8154. OVRO research on other solar systems is also supported by the Norris Planetary Origins Project and NASA Origins grant NAG5-9530.

\section{REFERENCES}

Aumann, H. H., et al. 1984, ApJ, 278, L23

Backman, D. E., \& Paresce, F. 1993, in Protostars and Planets III, ed. E. H. Levy \& J. I. Lunine (Tucson: Univ. Arizona Press), 1253

Beckwith, S. V. W., \& Sargent, A. I. 1993, in Protostars and Planets III, ed.

E. H. Levy \& J. I. Lunine (Tucson: Univ. Arizona Press), 521

Chini, R., Krügel, E., \& Kreysa, E. 1990, A\&A, 227, L5

Chini, R., Krügel, E., Shustov, B., Tutukov, A., \& Kreysa, E. 1991, A\&A, 252, 220

Cohen, M., Walker, R. G., Barlow, M. J., \& Deacon, J. R. 1992, AJ, 104, 1650
Dent, W. R. F., Walker, H. J., Holland, W. S., \& Greaves, J. S. 2000, MNRAS, 314, 702 (DWHG)

Gillett, F. C. 1986, in Light on Dark Matter, ed. F. P. Israel (Dordrecht: Reidel), 61

Goldreich, P., Tremaine, S., \& Borderies, N. 1986, AJ, 92, 490

Greaves, J. S., et al. 1998, ApJ, 506, L133

Gulliver, A. F., Hill, G., \& Adelman, S. J. 1994, ApJ, 429, L81

Hanninen, J., \& Porco, C. 1997, Icarus, 126, 1

Harvey, P. M., Wilking, B. A., \& Joy, M. 1984, Nature, 307, 441

Heinrichsen, I., Walker, H. J., \& Klaas, U. 1998, MNRAS, 293, L78 
Holland, W. S., et al. 1998, Nature, 392, 788 (H98)

Hubbard, W. B., Brahic, A., Sicardy, B., Elicer, L.-R., Roques, F., \& Vilas, F. 1986, Nature, 319, 636

Jura, M. 1991, ApJ, 383, L79

Koerner, D. W. 2001, in ASP Conf. Ser. 231, Tetons 4: Galactic Structure, Stars, and the Interstellar Medium, ed. C. E. Woodward, M. D. Bicay, \& J. M. Shull (San Francisco: ASP), 563

Koerner, D. W., Ressler, M. E., Werner, M. W., \& Backman, D. E. 1998, ApJ, 503, L83

Maeder, A., \& Meynet, G. 1988, A\&AS, 76, 411

Ozernoy, L. M., Gorkavyi, N. N., Mather, J. C., \& Taidakove, T. A. 2000, ApJ, 537, L147
Porco, C. C. 1991, Science, 253, 995

Schneider, G., et al. 1999, ApJ, 513, L127

Smith, B. A., et al. 1989, Science, 246, 1422

Smith, B. A., \& Terrile, R. J. 1984, Science, 226, 1421

Telesco, C. M., et al. 2000, ApJ, 530, 329

van der Bliek, N. A., Prusti, T., \& Waters, L. B. F. M. 1994, A\&A, 285, 229

Wyatt, M. C., Dermott, S. F., Telesco, C. M., Fisher, R. S., Grogan, K., Holmes, E. K., \& Piña, R. K. 1999, ApJ, 527, 918

Zuckerman, B., \& Becklin, E. E. 1993a, ApJ, 406, L25 . 1993b, ApJ, 414, 793 